\title{
POLE- AND ZERO-FREE REGIONS FOR ANALYTIC CONTINUED FRACTIONS
}

\author{
HANS-J. RUNCKEL
}

\begin{abstract}
By using well-known methods of analytic continued fraction theory, various types of zero-free regions are obtained for sequences of polynomials having complex coefficients and being defined by three-term recurrence relations. These results are related to recent investigations by $\mathrm{P}$. Henrici, E. B. Saff and R. S. Varga. As an application, zero-free sectors and stripes in $\mathbf{C}$ are obtained for the Bessel function $J_{v}$, where $\nu$ is complex. Analogous results are obtained for the Lommel polynomials associated with $J_{\nu}$.
\end{abstract}

1. Introduction. Consider the continued fraction

$$
w_{n}=\frac{A_{n}}{B_{n}}=\frac{a_{1}}{b_{1}}+\frac{a_{2}}{b_{2}}+\cdots+\frac{a_{n}}{b_{n}}, \quad n \in \mathbf{N},
$$

where $a_{n}, b_{n} \in \mathbf{C}$ and $a_{n} \neq 0$ for $n \in \mathbf{N}$. In the applications below, $a_{n}$ and $b_{n}$ are special analytic functions of $z$. Then (see [2, p. 20])

$$
\begin{aligned}
& B_{n}=b_{n} B_{n-1}+a_{n} B_{n-2}, \quad B_{0}=1, \quad B_{-1}=0, \quad n \in \mathbf{N}, \\
& A_{n}=b_{n} A_{n-1}+a_{n} A_{n-2}, \quad A_{0}=0, \quad A_{-1}=1, \quad n \in \mathbf{N} \text {, }
\end{aligned}
$$

hold.

Because $A_{n} B_{n-1}-B_{n} A_{n-1}=(-1)^{n-1} a_{1} a_{2} \cdots a_{n}, n \in \mathrm{N}, A_{n}$ and $B_{n}$ do not vanish simultaneously, and, hence, $w_{n}=\infty$ holds iff $B_{n}=0$. If a sequence $B_{n}$, $n \in \mathbf{N}$, is given which satisfies (2), then, after choosing $a_{1} \in \mathbf{C}, a_{1} \neq 0$, arbitrarily, $B_{n}$ can be considered as the $n$th denominator of a sequence of continued fractions $w_{n}=A_{n} / B_{n}$ of type (1).

We want to find conditions on $a_{n}, b_{n}$ which imply $w_{n} \neq \infty$ and, hence, $B_{n} \neq 0$ for $n \in \mathbf{N}$. Since, for $n \geqslant 2, w_{n}=a_{1} /\left(b_{1}+w_{n-1}^{*}\right)$ holds, where

$$
w_{n-1}^{*}=\frac{A_{n-1}^{*}}{B_{n-1}^{*}}=\frac{a_{2}}{b_{2}}+\frac{a_{3}}{b_{3}}+\cdots+\frac{a_{n}}{b_{n}},
$$

$A_{n}=0$ holds iff $B_{n-1}^{*}=0$ holds.

Hence, results concerning the nonvanishing of $B_{n}, n \in \mathbf{N}$ also yield results concerning the nonvanishing of $A_{n}, n \in \mathbf{N}$. In [4] the special case $a_{n}=-\alpha_{n} z$, $b_{n}=\beta_{n}+z, \alpha_{n}>0, \beta_{n}>0, n \in \mathbf{N}$, is considered, and zero-free parabolic regions are obtained for the polynomials $B_{n}(z)$. In [1] also zero-free parabolic regions are obtained for interpolation polynomials $B_{n}(z)$, where $a_{n}=-\alpha_{n} z_{n}, b_{n}=\beta_{n}+z_{n+1}$,

Received by the editors May 27, 1982 and, in revised form, March 6, 1985.

1980 Mathematics Subject Classification. Primary 30C15; Secondary 30B70, 33A40. 
$\alpha_{n}>0, \beta_{n}>0$, and $z_{n}=z-X_{n-1}$. Here $X_{n}, n \in \mathbf{N}$, are the real interpolation points. The results of [ 1 and 4] are generalized in [3] to sequences $B_{n}, n \in \mathbf{N}$, with complex $\alpha_{n}$ and $\beta_{n}$.

We first derive general conditions on $a_{n}, b_{n}$ which imply $B_{n} \neq 0$ for $n \in \mathbf{N}$ (Lemma 1). If $a_{n}$ and $b_{n}$ are special polynomials in $z$ of degree $\leqslant 2$, then Lemma 1 yields parabolic regions, sectors and stripes in $\mathbf{C}$ as zero-free regions for $B_{n}(z)$. In particular, zero-free regions of these types are obtained for the Bessel function $J_{\nu}$ and the corresponding Lommel polynomials, where $\nu$ is complex.

2. Main results. As in [1 and 3] the principal idea is to write (1) in the form $w_{N}=s_{1} \circ \cdots \circ s_{N}(0), N \in \mathbf{N}$, where $s_{n}(u):=a_{n} /\left(b_{n}+u\right), u \in \mathbf{C}, n \in \mathbf{N}$, and to determine a sequence of closed half-planes $H_{n} \subset \overline{\mathbf{C}}=\mathbf{C} \cup\{\infty\}$ with $0 \in H_{n}$, $n \in \mathbf{N}$, such that, for $n \in \mathbf{N}, D_{n}:=s_{n}\left(H_{n}\right)$ is a finite closed disk satisfying $D_{n} \subset H_{n-1}$ for $n \geqslant 2$. Then $w_{N} \in D_{1}$ and, hence, $w_{N} \neq \infty$ holds for $N \in \mathbf{N}$.

For $n \in \mathbf{N}$ define $H_{n}:=\left\{z \in \mathbf{C}: \operatorname{Re} e^{i \varphi_{n}}\left(z+d_{n}\right) \geqslant 0\right\} \cup\{\infty\}$, where $\varphi_{n} \in \mathbf{R}$ and $d_{n} \in \mathbf{C}$. Then $0 \in H_{n}$ if $\operatorname{Re} e^{i \varphi_{n}} d_{n} \geqslant 0$, and $D_{n}=s_{n}\left(H_{n}\right)$ is a finite disk iff $-b_{n} \notin H_{n}$; i.e., $\operatorname{Re} e^{i \varphi_{n}}\left(b_{n}-d_{n}\right)>0$.

Denote by $b_{n}^{*}$ the point symmetric to $-b_{n}$ with respect to $\partial H_{n}$; that is, $b_{n}^{*}=-d_{n}$ $+e^{-2 i \varphi_{n}}\left(\bar{b}_{n}-\bar{d}_{n}\right)$. Then because $s_{n}\left(-b_{n}\right)=\infty$, the center $\zeta_{n}$ of $D_{n}$ is given by

$$
\zeta_{n}=s_{n}\left(b_{n}^{*}\right)=\frac{a_{n}}{b_{n}+b_{n}^{*}}=\frac{a_{n} e^{i \varphi_{n}}}{2 \operatorname{Re} e^{i \varphi_{n}}\left(b_{n}-d_{n}\right)} .
$$

Since $s_{n}(\infty)=0 \in \partial D_{n}$, the radius $r_{n}$ of $D_{n}$ is given by

$$
r_{n}=\left|\zeta_{n}\right|=\left|a_{n}\right| / 2 \operatorname{Re} e^{i \varphi_{n}}\left(b_{n}-d_{n}\right) \text {. }
$$

Now $D_{n} \subset H_{n-1}$ holds iff $\operatorname{Re} e^{i \varphi_{n-1}}\left(\zeta_{n}+d_{n-1}\right)-r_{n} \geqslant 0$ holds $(n \geqslant 2)$. This is equivalent to

$$
\left|a_{n}\right| \leqslant \operatorname{Re} e^{i\left(\varphi_{n-1}+\varphi_{n}\right)} a_{n}+2\left(\operatorname{Re} e^{i \varphi_{n-1}} d_{n-1}\right)\left(\operatorname{Re} e^{i \varphi_{n}}\left(b_{n}-d_{n}\right)\right), \quad n \geqslant 2 .
$$

We have thus proved (see also [2, Chapter 4])

LEMMA 1. Assume that $a_{n}$ and $b_{n}$ in (1) or (2) satisfy (for suitable $\varphi_{n} \in \mathbf{R}$, $\left.d_{n} \in \mathbf{C}\right)$

$$
\operatorname{Re} e^{i \varphi_{n}}\left(b_{n}-d_{n}\right)>0, \quad \operatorname{Re} e^{i \varphi_{n}} d_{n} \geqslant 0, \quad n \in \mathbf{N}
$$

and

(4) $\left|a_{n}\right| \leqslant \operatorname{Re} e^{i\left(\varphi_{n-1}+\varphi_{n}\right)} a_{n}+2\left(\operatorname{Re} e^{i \varphi_{n-1}} d_{n-1}\right)\left(\operatorname{Re} e^{i \varphi_{n}}\left(b_{n}-d_{n}\right)\right), \quad n \geqslant 2$.

Then $B_{n} \neq 0$ for $n \in \mathbf{N}$. If (3) holds for $1 \leqslant n \leqslant N$ and (4) holds for $2 \leqslant n \leqslant N$, then $B_{n} \neq 0$ for $1 \leqslant n \leqslant N$.

REMARK. In (3) the condition $\operatorname{Re} e^{i \varphi_{1}}\left(b_{1}-d_{1}\right)>0$ can be replaced by the weaker condition $-b_{1} \notin D_{2}$-i.e.

$$
\left|2 b_{1} \operatorname{Re} e^{i \varphi_{2}}\left(b_{2}-d_{2}\right)+a_{2} e^{i \varphi_{2}}\right|>\left|a_{2}\right| \text {. }
$$

We now consider special cases of (1) and Lemma 1. 
THEOREM 1. Let

$$
\begin{gathered}
w_{n}(z)=\frac{A_{n}(z)}{B_{n}(z)}=\frac{1}{1}+\frac{c_{1} c_{2} z}{1}+\frac{c_{2} c_{3} z}{1}+\cdots+\frac{c_{n-1} c_{n} z}{1}, \\
B_{n}(z)=B_{n-1}(z)+c_{n-1} c_{n} z B_{n-2}(z), \quad n \geqslant 1, \quad B_{0}=1, \quad B_{-1}=0,
\end{gathered}
$$

where $c_{n} \in \mathbf{C}, c_{n}=\left|c_{n}\right| e^{i \psi_{n}} \neq 0, \alpha \leqslant \psi_{n}<\beta, \beta-\alpha<\pi, n \in \mathbf{N}$.

(a) Then $B_{n}(z) \neq 0$ for $n \geqslant 1$ and all $z=|z| e^{i \vartheta}$ satisfying $-\pi-2 \alpha<\vartheta \leqslant \pi-2 \beta$.

(b) If $I:=\{\varphi \in \mathbf{R}: \beta-\pi / 2 \leqslant \varphi<\alpha+\pi / 2\}$,

$$
r(\varphi):=\inf _{n \geqslant 2} \operatorname{Re}\left(e^{i \varphi} / c_{n-1}\right) \operatorname{Re}\left(e^{i \varphi} / c_{n}\right) \text { for } \varphi \in I,
$$

and if, for $r \geqslant 0, P(r, \varphi):=\left\{z \in \mathbf{C}:|z| \leqslant \operatorname{Re} e^{2 i \varphi} z+r / 2\right\}$, then $B_{n}(z) \neq 0$ for $n \geqslant 1$ and all $z \in \bigcup_{\varphi \in I} P(r(\varphi), \varphi)$.

Proof. Put $a_{1}=1, a_{n}=c_{n-1} c_{n} z, n \geqslant 2, b_{n}=1, n \geqslant 1$, in (1) and apply Lemma 1. (a) Choose $d_{n}=0$ and $e^{i \varphi_{n}}=e^{-i \vartheta / 2}\left|c_{n}\right| / c_{n}, n \geqslant 1$. Then (4) is trivially satisfied, and (3) reduces to $\operatorname{Re} e^{i \vartheta / 2} c_{n}>0, n \geqslant 1$, which is satisfied if $-\pi / 2<\psi_{n}+\vartheta / 2<$ $\pi / 2, n \geqslant 1$, or $-\pi-2 \alpha<\vartheta \leqslant \pi-2 \beta$.

(b) Choose $d_{n}=1 / 2$ and $e^{i \varphi_{n}}=e^{i \varphi}\left|c_{n}\right| / c_{n}, n \geqslant 1$. Then (3) and (4) reduce to $\operatorname{Re} e^{i \varphi} / c_{n}>0$ and $|z| \leqslant \operatorname{Re} e^{2 i \varphi} z+\frac{1}{2} \operatorname{Re}\left(e^{i \varphi} / c_{n-1}\right) \operatorname{Re}\left(e^{i \varphi} / c_{n}\right) . \operatorname{Re} e^{i \varphi} / c_{n}>0$ is satisfied if $-\pi / 2<\varphi-\psi_{n}<\pi / 2, n \geqslant 1$, or $\beta-\pi / 2 \leqslant \varphi<\alpha+\pi / 2$.

THEOREM 2. Let

$$
\begin{gathered}
w_{n}(z)=\frac{A_{n}(z)}{B_{n}(z)}=\frac{1}{1+e_{1} z}+\frac{c_{1} c_{2} z^{2}}{1+e_{2} z}+\frac{c_{2} c_{3} z^{2}}{1+e_{3} z}+\cdots+\frac{c_{n-1} c_{n} z^{2}}{1+e_{n} z}, \\
B_{n}(z)=\left(1+e_{n} z\right) B_{n-1}(z)+c_{n-1} c_{n} z^{2} B_{n-2}(z), \quad n \geqslant 1, \quad B_{0}=1, \quad B_{-1}=0,
\end{gathered}
$$

where $e_{n}, c_{n} \in \mathbf{C}$ and $c_{n}=\left|c_{n}\right| e^{i \psi_{n}} \neq 0, \alpha \leqslant \psi_{n}<\beta, \beta-\alpha<\pi$ for $n \in \mathbf{N}$.

(a) If $\operatorname{Re}\left(e_{n} / c_{n}\right) \geqslant 0(\leqslant 0), n \geqslant 1$, then $B_{n}(z) \neq 0$ for $n \geqslant 1$ and all $z=|z| e^{i \vartheta}$ satisfying $-\alpha-\pi / 2<\vartheta \leqslant-\beta+\pi / 2(-\alpha+\pi / 2<\vartheta \leqslant-\beta+3 \pi / 2)$.

(b) If $e_{n}=0, n \geqslant 1$, and $I, r(\varphi)$ are the same as in Theorem 1, and if, for $r \geqslant 0$, $S(r, \varphi):=\left\{z \in \mathbf{C}:\left|\operatorname{Im} e^{i \varphi} z\right| \leqslant \sqrt{r} / 2\right\}$, then $B_{n}(z) \neq 0$ for $n \geqslant 1$ and all $z \in$ $\cup_{\varphi \in I} S(r(\varphi), \varphi)$.

Proof. Put $a_{1}=1, a_{n}=c_{n-1} c_{n} z^{2}, n \geqslant 2, b_{n}=1+e_{n} z, n \geqslant 1$, in (1) and apply Lemma 1. (a) Choose $d_{n}=e_{n} z$ and $e^{i \varphi_{n}}=e^{-i \vartheta}\left|c_{n}\right| / c_{n}, n \geqslant 1$. Then (4) is trivially satisfied, and (3) reduces to $\operatorname{Re}\left(e_{n} / c_{n}\right) \geqslant 0$ and $\operatorname{Re} z c_{n}>0, n \geqslant 1$, which is satisfied if $-\pi / 2<\psi_{n}+\vartheta<\pi / 2, n \geqslant 1$, or $-\alpha-\pi / 2<\vartheta \leqslant-\beta+\pi / 2$. Similarly, $e^{i \varphi_{n}}=$ $-e^{-i{ }^{*}}\left|c_{n}\right| / c_{n}$ yields the conditions $\operatorname{Re}\left(e_{n} / c_{n}\right) \leqslant 0$ and $\operatorname{Re}\left(-z c_{n}\right)>0$ or $-\pi / 2<-\pi$ $+\psi_{n}+\vartheta<\pi / 2, n \geqslant 1$, which is satisfied if $-\alpha+\pi / 2<\vartheta \leqslant-\beta+3 \pi / 2$.

(b) Put $d_{n}=1 / 2$ and $e^{i \varphi_{n}}=e^{i \varphi}\left|c_{n}\right| / c_{n}$. Then (4) and (3) reduce to

$$
\left|z^{2}\right| \leqslant \operatorname{Re}\left(e^{i \varphi} z\right)^{2}+\operatorname{Re}\left(e^{i \varphi} / c_{n-1}\right) \operatorname{Re}\left(e^{i \varphi} / c_{n}\right) / 2
$$

or $2\left|\operatorname{Im} e^{i \varphi} z\right| \leqslant\left(\operatorname{Re}\left(e^{i \varphi} / c_{n-1}\right) \operatorname{Re}\left(e^{i \varphi} / c_{n}\right)\right)^{1 / 2}, \quad n \geqslant 2$, and $\operatorname{Re} e^{i \varphi} / c_{n}>0, n \geqslant 1$, which is satisfied if $-\pi / 2<\varphi-\psi_{n}<\pi / 2, n \geqslant 1$ or $\beta-\pi / 2 \leqslant \varphi<\alpha+\pi / 2$. 
THEOREM 3. Let

$$
\begin{gathered}
w_{n}(z)=\frac{A_{n}(z)}{B_{n}(z)}=\frac{1}{g_{1} z+h_{1}}+\frac{1}{g_{2} z+h_{2}}+\cdots+\frac{1}{g_{n} z+h_{n}}, \\
B_{n}(z)=\left(g_{n} z+h_{n}\right) B_{n-1}(z)+B_{n-2}(z), \quad n \geqslant 1, \quad B_{0}=1, \quad B_{-1}=0,
\end{gathered}
$$

where $g_{n}, h_{n} \in \mathbf{C}, g_{n}=\left|g_{n}\right| e^{i \psi_{n}} \neq 0, \alpha \leqslant \psi_{n}<\beta, \beta-\alpha<\pi$ for $n \in \mathbf{N}$.

(a) If $\operatorname{Re} h_{n} \geqslant 0(\leqslant 0), n \geqslant 1$, then $B_{n}(z) \neq 0$ for $n \geqslant 1$ and all $z=|z| e^{i \vartheta} \neq 0$ satisfying $-\alpha-\pi / 2<\vartheta \leqslant-\beta+\pi / 2(-\alpha+\pi / 2<\vartheta \leqslant-\beta+3 \pi / 2)$.

(b) If $h_{n}=0, n \geqslant 1$,

$$
\begin{gathered}
J:=\{\varphi \in \mathbf{R}:-\alpha-\pi / 2<\varphi \leqslant-\beta+\pi / 2\}, \\
s(\varphi):=\inf _{n \geqslant 2} \operatorname{Re}\left(e^{i \varphi} g_{n-1}\right) \operatorname{Re}\left(e^{i \varphi} g_{n}\right)
\end{gathered}
$$

for $\varphi \in J$, and if

$$
D(0, \varphi):=\left\{z \in \mathbf{C}: \operatorname{Im} e^{-i \varphi} z=0\right\}
$$

and

$$
D(s, \varphi):=\left\{z \in \mathbf{C}:\left|z-i e^{i \varphi} / \sqrt{s}\right| \geqslant 1 / \sqrt{s}\right\} \cap\left\{z \in \mathbf{C}:\left|z+i e^{i \varphi} / \sqrt{s}\right| \geqslant 1 / \sqrt{s}\right\}
$$

for $s>0$, then $B_{n}(z) \neq 0$ for $n \geqslant 1$ and all $z \in \cup_{\varphi \in J} D(s(\varphi), \varphi) \backslash\{0\}$.

Proof. Put $a_{n}=1, b_{n}=g_{n} z+h_{n}, n \geqslant 1$, in (1) and apply Lemma 1.

(a) Choose $d_{n}=0, n \geqslant 1$. Then (3) and (4) reduce to $\operatorname{Re} e^{i \varphi_{n}}\left(g_{n} z+h_{n}\right)>0$, $n \geqslant 1$, and $1 \leqslant \cos \left(\varphi_{n-1}+\varphi_{n}\right), n \geqslant 2$. This holds if $\varphi_{n}=0, \operatorname{Re} h_{n} \geqslant 0$ and $\operatorname{Re} z g_{n}$ $>0$ or $-\pi / 2<\vartheta+\psi_{n}<\pi / 2, n \geqslant 1$, which is satisfied if $-\alpha-\pi / 2<\vartheta \leqslant-\beta+$ $\pi / 2$. Similarly, $\varphi_{n}=\pi, n \geqslant 1$, yields the conditions $\operatorname{Re} h_{n} \leqslant 0$ and $\operatorname{Re}\left(-z g_{n}\right)>0$ or $-\pi / 2<-\pi+\vartheta+\psi_{n}<\pi / 2, n \geqslant 1$, which is satisfied if $-\alpha+\pi / 2<\vartheta \leqslant-\beta+$ $3 \pi / 2$.

(b) Put $d_{n}=z g_{n} / 2$ and $e^{i \varphi_{n}}=e^{i \varphi}|z| / z, n \geqslant 1$. Then (4) and (3) reduce to

$$
1 \leqslant \operatorname{Re}\left(\bar{z} e^{i \varphi} /|z|\right)^{2}+|z|^{2} \operatorname{Re}\left(e^{i \varphi} g_{n-1}\right) \operatorname{Re}\left(e^{i \varphi} g_{n}\right) / 2, \quad n \geqslant 2,
$$

and

$$
\operatorname{Re}\left(e^{i \varphi} g_{n}\right)>0 \text { or }-\dot{\pi} / 2<\varphi+\psi_{n}<\pi / 2, \quad n \geqslant 1,
$$

which is satisfied if $-\alpha-\pi / 2<\varphi \leqslant-\beta+\pi / 2$. Finally, one verifies that for $s \geqslant 0$,

$$
\left\{z \in \mathrm{C}:|z|^{2} \leqslant \operatorname{Re}\left(z e^{-i \varphi}\right)^{2}+|z|^{4} s / 2\right\}=D(s, \varphi) .
$$

REMARK. If the assumptions in Theorems $1-3$ hold for $1 \leqslant n \leqslant N$ only, then the conclusions are valid for $1 \leqslant n \leqslant N$, provided $r(\varphi), s(\varphi)$ are replaced by

$$
r_{N}(\varphi):=\min _{2 \leqslant n \leqslant N} \operatorname{Re}\left(\frac{e^{i \varphi}}{c_{n-1}}\right) \operatorname{Re}\left(\frac{e^{i \varphi}}{c_{n}}\right) \text { for } \varphi \in I,
$$

and

$$
s_{N}(\varphi):=\min _{2 \leqslant n \leqslant N} \operatorname{Re}\left(e^{i \varphi} g_{n-1}\right) \operatorname{Re}\left(e^{i \varphi} g_{n}\right) \quad \text { for } \varphi \in J
$$


3. Application to Bessel functions and Lommel polynomials. Let $J_{\nu}(z)=$ $(z / 2)^{\nu} \phi_{\nu}(z)$ be the $\nu$ th Bessel function, where

$$
\phi_{\nu}(z):=\sum_{n=0}^{\infty}(-1)^{n} \frac{(z / 2)^{2 n}}{n ! \Gamma(\nu+n+1)}, \quad \nu, z \in \mathbf{C}, \nu \neq-1,-2, \ldots
$$

Then (see also [5, Chapter III])

$$
\begin{gathered}
\phi_{\nu}^{\prime}(z)=-(z / 2) \phi_{\nu+1}(z), \\
\phi_{\nu}(z)=(\nu+1) \phi_{\nu+1}(z)-(z / 2)^{2} \phi_{\nu+2}(z), \\
z \phi_{\nu}^{\prime \prime}(z)+(2 \nu+1) \phi_{\nu}^{\prime}(z)+z \phi_{\nu}(z)=0 .
\end{gathered}
$$

Here (7) implies that all $\phi_{\nu}$ have simple zeros. Then (5) shows that $\phi_{\nu}, \phi_{\nu+1}$ have no common zeros. Using [2, Theorem 5.16], we obtain from (6) that

$$
\frac{\phi_{\nu+1}(z)}{\phi_{\nu}(z)}=\frac{1}{\nu+1}-\frac{(z / 2)^{2}}{\nu+2}-\frac{(z / 2)^{2}}{\nu+3}-\cdots,
$$

where the infinite continued fraction is uniformly convergent on each compact set of C avoiding the poles of $\phi_{\nu+1} / \phi_{\nu}$-i.e., the zeros of $\phi_{\nu}$. After applying an equivalence transformation to (8), we obtain

$$
\frac{(\nu+1) \phi_{\nu+1}(z)}{\phi_{\nu}(z)}=\frac{1}{1}+\frac{c_{1} c_{2} z^{2}}{1}+\frac{c_{2} c_{3} z^{2}}{1}+\cdots,
$$

where $c_{n}=1 / 2 i(\nu+n), n \geqslant 1$. Applying Theorem 2 to the sequence of approximants of (9) or, equivalently, of (8) yields

THEOREM 4. If $\nu=\nu_{1}+i \nu_{2}, \nu_{1}, \nu_{2} \in \mathbf{R}$, such that $0 \leqslant \arg (\nu+1)<\pi$, and if

$$
\begin{gathered}
B_{n, \nu}(z)=(\nu+n) B_{n-1, \nu}(z)-(z / 2)^{2} B_{n-2, \nu}(z), \quad n \geqslant 1, \\
B_{0, \nu}=1, \quad B_{-1, \nu}=0,
\end{gathered}
$$

then $B_{n, v}(z) \neq 0$ for $n \geqslant 1$ and

(a) all $z=|z| e^{i \vartheta}$ satisfying $\arg (\nu+1)<\vartheta \leqslant \pi$ or $\arg (\nu+1)+\pi<\vartheta \leqslant 2 \pi$,

(b) all $z \in \mathbf{C}$ satisfying $|\operatorname{Im} z| \leqslant \nu_{2}$, provided $\nu_{2}>0$. Also,

(c) if $0 \leqslant \arg (\nu+1)<\pi / 2$, then, in addition to (a) and (b), $B_{n, \nu}(z) \neq 0$ for $n \geqslant 1$ and all $z \in \mathbf{C}$ satisfying $(\operatorname{Re} z)^{2} \leqslant\left(\nu_{1}+1\right)\left(\nu_{1}+2\right)$.

Because $B_{n, \bar{\nu}}(z)=\overline{B_{n, \nu}(\bar{z})}$, the zeros of $B_{n, \bar{\nu}}$ are the conjugates of the zeros of $B_{n, \nu}$.

Proof. With the notations of Theorem 2 we obtain $e_{n}=0, n \geqslant 1, \alpha=$ $-\arg (\nu+1)-\pi / 2 \leqslant \psi_{n}<\beta=-\pi / 2$ and $\beta-\alpha=\arg (\nu+1)$. Then (a) follows from Theorem 2(a). Furthermore,

$$
I=\{\varphi:-\pi \leqslant \varphi<-\arg (\nu+1)\}
$$

and

$$
r(\varphi)=\inf _{n \geqslant 2} 4 \operatorname{Re}\left(e^{i \varphi} i(\nu+n-1)\right) \operatorname{Re}\left(e^{i \varphi} i(\nu+n)\right) \quad \text { for } \varphi \in I .
$$

Then Theorem 2(b) implies $B_{n, \nu}(z) \neq 0$ for $n \geqslant 1$ and all $z \in \cup_{\varphi \in I} S(r(\varphi), \varphi)$. Choosing especially $\varphi=-\pi$ and $\varphi=-\pi / 2$ yields (b) and (c). 
COROllaRY 1. If $\nu=\nu_{1}+i \nu_{2}, \nu_{1}, \nu_{2} \in \mathbf{R}$, such that $0 \leqslant \arg (\nu+1)<\pi$, then $\phi_{\nu}(z) \neq 0$ for

(a) all $z=|z| e^{i \vartheta}$ satisfying $\arg (\nu+1)<\vartheta<\pi$ or $\arg (\nu+1)+\pi<\vartheta<2 \pi$,

(b) all $z \in \mathbf{C}$ satisfying $|\operatorname{Im} z|<\nu_{2}$, provided $\nu_{2}>0$. Also,

(c) if $0 \leqslant \arg (\nu+1)<\pi / 2$, then, in addition to (a) and (b), $\phi_{\nu}(z) \neq 0$ for all $z \in \mathrm{C}$ satisfying $(\operatorname{Re} z)^{2}<\left(\nu_{1}+1\right)\left(\nu_{1}+2\right)$. (See also [5, p. 486] for the special case $\nu+1>0$.)

Because $\phi_{\bar{\nu}}(z)=\overline{\phi_{\nu}(\bar{z})}$, the zeros of $\phi_{\bar{\nu}}$ are the conjugates of the zeros of $\phi_{\nu}$.

REMARK. Because of the asymptotic formula [5, p. 199]

$$
J_{\nu}(z) \sim(2 / \pi z)^{1 / 2} \cos (z-\nu \pi / 2-\pi / 4),
$$

valid for $z \rightarrow \infty,-\pi<\arg z<\pi$, the "large" zeros of $J_{\nu}$ are near the lines $\operatorname{Im} z=\operatorname{Im} \nu \pi / 2$ for $\operatorname{Re} z>0$ or $\operatorname{Im} z=-\operatorname{Im} \nu \pi / 2$ for $\operatorname{Re} z<0$, provided $\operatorname{Im} \nu>0$. See also [5, pp. 495-497].

Next, the Lommel polynomials $g_{m, \nu}(z)$, being related to $B_{m, \nu}(z)$, are defined as follows [5, p. 303]:

$$
\begin{aligned}
& g_{m, \nu}(z):= \sum_{0 \leqslant n \leqslant m / 2}(-1)^{n}\left(\begin{array}{c}
m-n \\
n
\end{array}\right) z^{n} \frac{\Gamma(\nu+m-n+1)}{\Gamma(\nu+n+1)}, \\
& \nu, z \in \mathbf{C}, \nu \neq-1,-2, \ldots .
\end{aligned}
$$

Then

(10)

$$
g_{m, \nu}(z)=(\nu+m) g_{m-1, \nu}(z)-z g_{m-2, \nu}(z), \quad m \geqslant 1, \quad g_{0, \nu}=1, \quad g_{-1, \nu}=0 .
$$

Therefore $g_{m, \nu}$ and $g_{m-1, \nu}$ have no common zeros. Furthermore by [5, p. 302], $\lim _{m \rightarrow \infty} g_{m, \nu}\left(z^{2} / 4\right) / \Gamma(\nu+m+1)=\phi_{\nu}(z)$ uniformly on each compact set in $\mathbf{C}$. (10) yields

$$
\frac{g_{m-1, \nu}(z)}{g_{m, \nu}(z)}=\frac{1}{\nu+m}-\frac{z}{\nu+m-1}-\cdots-\frac{z}{\nu+1}
$$

or, after applying an equivalence transformation,

$$
\frac{(\nu+m) g_{m-1, \nu}(z)}{g_{m, \nu}(z)}=\frac{1}{1}+\frac{c_{1} c_{2} z}{1}+\cdots+\frac{c_{m-1} c_{m} z}{1},
$$

where $c_{n}=1 / i(\nu+m+1-n), 1 \leqslant n \leqslant m$.

Applying Theorem 1 to (11) yields

THEOREM 5. If $\nu=\nu_{1}+i \nu_{2}, \nu_{1}, \nu_{2} \in \mathbf{R}$, such that $0 \leqslant \arg (\nu+1)<\pi$, then for each $m \in \mathbf{N}, g_{m, \nu}(z) \neq 0$ for

(a) all $z=|z| e^{i \vartheta}$ satisfying $2 \arg (\nu+1)<\vartheta<2 \pi+2 \arg (\nu+m)$,

(b) all $z \in \mathbf{C}$ satisfying $|z| \leqslant \operatorname{Re} z+\nu_{2}^{2} / 2$. Also,

(c) if $0 \leqslant \arg (\nu+1)<\pi / 2$, then, in addition to (a), (b), $g_{m, \nu}(z) \neq 0$ for all $z \in \mathbf{C}$ satisfying $|z| \leqslant-\operatorname{Re} z+\left(\nu_{1}+1\right)\left(\nu_{1}+2\right) / 2$.

Because $g_{m, \bar{\nu}}(z)=\overline{g_{m, \nu}(\bar{z})}$, the zeros of $g_{m, \bar{\nu}}$ are the conjugates of the zeros of $g_{m, \nu}$. 
Proof. With the notation of Theorem 1 , we obtain, for $1 \leqslant n \leqslant m, \alpha=$ $-\arg (\nu+1)-\pi / 2 \leqslant \psi_{n} \leqslant \beta=-\arg (\nu+m)-\pi / 2$. Then (a) follows from Theorem 1(a). Furthermore,

$$
I=\{\varphi:-\arg (\nu+m)-\pi<\varphi<-\arg (\nu+1)\}
$$

and

$$
r(\varphi)=\min _{2 \leqslant n \leqslant m} \operatorname{Re}\left(e^{i \varphi} i(\nu+n-1)\right) \operatorname{Re}\left(e^{i \varphi} i(\nu+n)\right) \quad \text { for } \varphi \in I .
$$

Then Theorem 1(b) implies $g_{m, v}(z) \neq 0$ for all $z \in \bigcup_{\varphi \in I} P(r(\varphi), \varphi)$. Choosing especially $\varphi=-\pi$ and $\varphi=-\pi / 2$ yields (b) and (c).

REMARK. Using (9) and applying Theorem 2 to the infinite continued fraction (with arbitrary $c_{\nu}, e_{\nu} \in \mathbf{C}, c_{\nu} \neq 0,1 \leqslant \nu \leqslant n$ )

$$
\begin{gathered}
\frac{1}{1+e_{1} z}+\frac{c_{1} c_{2} z^{2}}{1+e_{2} z}+\cdots+\frac{c_{n-1} c_{n} z^{2}}{1+e_{n} z+c_{n} c_{n+1} z^{2}(\nu+1) \phi_{\nu+1}(z) / \phi_{\nu}(z)} \\
=\frac{(\nu+1) c_{n} c_{n+1} z^{2} A_{n-1}(z) \phi_{\nu+1}(z)+A_{n}(z) \phi_{\nu}(z)}{(\nu+1) c_{n} c_{n+1} z^{2} B_{n-1}(z) \phi_{\nu+1}(z)+B_{n}(z) \phi_{\nu}(z)},
\end{gathered}
$$

one can obtain information on the location of zeros of

$$
(\nu+1) c_{n} c_{n+1} z^{2} B_{n-1}(z) \phi_{\nu+1}(z)+B_{n}(z) \phi_{\nu}(z)
$$

or, using (5), $B_{n}(z) \phi_{\nu}(z)-2(\nu+1) c_{n} c_{n+1} z B_{n-1}(z) \phi_{\nu}^{\prime}(z)$.

\section{REFERENCES}

1. P. Henrici, Note on a theorem of Saff and Varga, Padé and Rational Approximation, Theory and Applications (E. B. Saff and R. S. Varga, eds.), Academic Press, New York, 1977.

2. W. B. Jones and W. J. Thron, Continued fractions, analytic theory and applications, Addison-Wesley, London. 1980.

3. H. J. Runckel, Zero-free parabolic regions for polynomials with complex coefficients, Proc. Amer. Math. Soc. 88 (1983), 299-304.

4. E. B. Saff and R. S. Varga, Zero-free parabolic regions for sequences of polynomials, SIAM J. Math. Anal. 7 (1976), 344-357.

5. (i. N. Watson, A treatise on the theory of Bessel functions, 2nd ed., Cambridge Univ. Press, 1966.

abthil.ung Mathematik IV, Universität Ulm, D-7900 Ulm (Donau), Oberer, Eselsberg, Federal Ri:PUBBIIC OF GeRMANY 